\title{
Refletividade em Espelhos de Bragg de AlGaAsSb/AlAsSb sobre InP
}

\author{
Reflectivity in Bragg Mirrors of AlGaAsSb/AlAsSb on InP
}

\author{
Dari de Oliveira Toginho Filho ${ }^{1}$; Ivan Frederico Lupiano Dias'; \\ José Leonil Duarte²; Sidney Alves Lourenço²; Luiz Carlos Poças²; Edson Laureto²; \\ Bernard Nabet ${ }^{3}$; Jean C.Harmand ${ }^{4}$
}

\begin{abstract}
Resumo
Analisamos neste trabalho, a refletividade de um espelho de Bragg composto por materiais da família do antimônio (AlGaAsSb/AlAsSb) dopado com telúrio. As amostras foram preparadas através da técnica de epitaxia por feixe molecular MBE (Molecular Beam Epitaxy) e as medidas de refletividade pela técnica de espectroscopia de infravermelho por transformada de Fourier FTIR (Fourier Transform InfraRed). Para discussão das propriedades do espelho de Bragg comparamos a refletividade obtida experimentalmente e uma simulação baseada no formalismo matricial.

Palavras-chave: semicondutores, refletividade, Espelho de Bragg, AlGaAsSb, AlAsSb.
\end{abstract}

\begin{abstract}
The reflectivity of a Bragg mirror composed by materials of the antimony family (AlGaAsSb/AlAsSb), doped with tellurium, is analyzed in this work. The sample was prepared by molecular beam epitaxy (MBE) and the reflectivity was measured by Fourier Transform Infra-Red Spectroscopy (FTIR). In order to discuss the Bragg mirror properties, the experimental reflectivity is compared to a simulation of the reflectivity based on the matrix formalism.
\end{abstract}

Key words: semiconductors, reflectance, Bragg mirror, AlGaAsSb, AlAsSb

\section{Introdução}

Espelhos de Bragg-DBR (Distributed Bragg Reflectors) são estruturas preparadas com a superposição de múltiplas camadas de materiais semicondutores (ou dielétricos) visando a máxima reflexão em um determinado comprimento de onda $(\lambda)$. As espessuras das diferentes camadas de materiais são definidas a partir do índice de refração pela expressão: $\mathrm{d}=\lambda / 4 n$, sendo $n$ o índice de refração e $\lambda$ o comprimento de onda desejado. Estas estruturas tem sido muito estudadas recentemente devido ao seu emprego na fabricação de dispositivos optoeletrônicos como os lasers de emissão pela superfície VCSEL (Vertical Cavity Surface Emiting Lasers) (SALE, 1995), (NAKAGAWA et al., 2001).

\footnotetext{
${ }^{1}$ Departamento de Física, Universidade Estadual de Londrina, Londrina/Pr, Brazil. darit@uel.br

${ }^{2}$ Departamento de Física, Universidade Estadual de Londrina, Londrina/Pr, Brazil

${ }^{3}$ CNET, Laboratoire CDP, 196 rue H. Ravera, 92220 Bagneux, France.

${ }^{4}$ CNRS, Laboratoire de Photonique et de Nanostructures, Route de Nozay, 91460 Marcoussis, France.
} 
A performance de um laser vertical é limitada principalmente pela disponibilidade e qualidade dos materiais que são empregados na fabricação dos espelhos de Bragg que formam a microcavidade Fabry-Perot necessária a sua operação. Para obter VCSELs operando a baixas correntes limiar (threshold), a temperatura ambiente, é necessário a preparação de espelhos de Bragg com refletividade superior a $99 \%$ e baixa resistência elétrica nas interfaces entre os diferentes materiais da estrutura (SALE, 1995). Considerando-se estas condições dadas pela refletividade e resistência elétrica, as propriedades mais importantes para a escolha dos diferentes sistemas de materiais que tem potencial para serem empregados em DBRs são: a) alto contraste entre os índices de refração $(\Delta n)$ dos diferentes materiais que compõem as camadas do espelho de Bragg de modo a obter a alta refletividade requerida; b) baixa resistência elétrica em série em baixos níveis de concentração de dopantes de modo a possibilitar baixas correntes de limiar e baixa absorção por portadores livres.

A primeira condição (grande contraste do índice de refração) implica na escolha de sistemas de materiais com grande descontinuidade entre as bandas de energia proibida $\left(\Delta \mathrm{E}_{\mathrm{g}}\right)$. Neste caso é necessário aperfeiçoar a injeção de portadores de carga nas interfaces entre os diferentes materiais que compõe a estrutura, utilizando altas concentrações de dopantes (entre $1 \times 10^{18} \mathrm{~cm}^{-3}$ e $1 \times 10^{19} \mathrm{~cm}^{-3}$ ) e/ou introduzir aperfeiçoamentos que permitam uma passagem mais efetiva da corrente como a modulação da dopagem ou a variação gradual da composição das ligas dos materiais envolvidos na região da interface (SALE, 1995). A condição de baixa resistência elétrica pode ser considerada, a priori, pela escolha de materiais com pequenas descontinuidade entre as bandas de energia proibida. Esta condição depende da distribuição da descontinuidade total das bandas de energia proibida ("offset") para a banda de condução $\left(\Delta \mathrm{E}_{\mathrm{C}}\right)$, e para a banda de valência $\left(\Delta \mathrm{E}_{\mathrm{v}}\right)$. Um "offset" pequeno para a banda de condução implica em baixa resistência à passagem dos elétrons nas interfaces, entre os materiais componentes de espelhos de Bragg dopados tipo-n. Entretanto, esta é uma condição excludente para a baixa resistência elétrica em espelhos de Bragg dopados tipo-p, fabricados com os mesmos materiais. Um outro dado importante é que em materiais com menor descontinuidade entre a banda de energia, temos um pequeno contraste de índices de refração, sendo necessário um grande número de interfaces para a obtenção da alta refletividade requerida para o funcionamento do laser. Isto traz dificuldades suplementares para a preparação de amostras e para os processos tecnológicos utilizados na fabricação do laser.

Espelhos de Bragg de GaAs/AlAs tem sido empregados com sucesso na fabricação de VCSELs para intervalos de pequenos comprimentos de onda $(0,8 \mu \mathrm{m}$ a $1,0 \mu \mathrm{m})($ EBELING, 1995), (GIEHLER et al., 2002). Este sistema apresenta uma grande descontinuidade de bandas de energia e um grande contraste de índices de refração $(\Delta n \cong 0,5)$ (SALE, 1995). Para o desenvolvimento de espelhos de Bragg em intervalos de maiores comprimentos de onda $(1,3 \mu \mathrm{m}$ a $1,6 \mu \mathrm{m})$ - janela de baixa dispersão e baixa atenuação para a transmissão por fibras ópticas - um sistema com parâmetro de rede "acordado" a substratos de InP é o mais desejado devido ao atual estágio de maturidade tecnológica no processamento deste material (HARMAND et al., 1995). Vários sistemas de materiais semicondutores tem sido experimentados (ex: InGaAsP/InP, AlGaInAs/ AlInAs, AlGaAsSb/AlAsSb) (DIAS et al., 1998) contudo, até o momento, existem em todos eles problemas não resolvidos. Em sistemas de InGaAsP/ InP e AlGaInAs/AlInAs o contraste de índice de refração é pequeno $(\Delta n \cong 0,3)$ o que exige a preparação de estruturas, para os espelhos de Bragg, com um grande número de períodos (30 a 50) para se atingir $99 \%$ de refletividade. O sistema $\mathrm{AlGaAsSb} /$ $\mathrm{AlAsSb}$ apresenta um grande contraste entre os índices de refração $(\Delta n \sim 0.54)$, entretanto, é um sistema pouco estudado e que apresenta ainda, devido a isto, dificuldades para a preparação de cristais livres 
de deslocações (HARMAND et al., 1997; (DIAS et al., 1997, 1998). Portanto, na faixa de grandes comprimentos de onda, não está ainda estabelecido qual sistema de materiais é mais adequado para a preparação de microcavidades para VCSELs.

Neste trabalho analisaremos a refletividade de um espelho de Bragg composto por materiais do sistema $\mathrm{AlGaAsSb} / \mathrm{AlAsSb}$, dopado com telúrio, preparado pela técnica de crescimento epitaxial por feixe molecular MBE (Molecular Beam Epitaxy). Faremos uma revisão do comportamento de uma onda eletromagnética plana se propagando através de um meio material e da dependência do índice de refração e do vetor de onda em função das características da onda eletromagnética e das propriedades do meio material. Para a discussão dos parâmetros estruturais, definidos na preparação de espelhos de Bragg (espessura das diferentes camadas, composição das ligas), e das propriedades ópticas dos materiais que compõem o espelho(AlGaAsSb/AlAsSb), desenvolvemos uma comparação entre a refletividade obtida experimentalmente e uma simulação baseada no formalismo matricial. Obtivemos a refletividade experimentalmente pela técnica de espectroscopia do infravermelho por transformada de Fourier. A simulação baseada no formalismo matricial foi desenvolvida com o aplicativo Mathcad.

\section{Propriedades ópticas}

A propagação de uma onda eletromagnética plana através de um meio material pode ser descrita através das equações de onda para o campo elétrico $\mathbf{E}$ ou para a indução magnética $\mathbf{B}$. As equações de onda, obtidas a partir das equações de Maxwell no sistema MKS são descritas por (REITZ, MILFORD; CHRISTY, 1982):

$$
\begin{aligned}
& \nabla^{2} \mathbf{E}-\mu \varepsilon \varepsilon_{0} \frac{\partial^{2} \mathbf{E}}{\partial t^{2}}=0 \\
& \nabla^{2} \mathbf{B}-\mu \varepsilon \varepsilon_{0} \frac{\partial^{2} \mathbf{B}}{\partial t^{2}}=0
\end{aligned}
$$

sendo $\mathbf{E}$ o campo elétrico, $\mathbf{B}$ a indução magnética, $\nabla$ a derivada direcional, $\boldsymbol{\varepsilon}$ a função dielétrica, $\varepsilon_{0}$ a permissividade elétrica do vácuo e $\mu$ a permeabilidade magnética do meio material. $\mathrm{O}$ campo elétrico está relacionado ao deslocamento elétrico através da relação $\mathbf{D}=\varepsilon \mathbf{E}$, e a indução magnética está relacionada ao vetor intensidade magnética pela relação $\mathbf{B}=\mu \mathbf{H}$. No sistema MKS $\varepsilon_{0}=8,85 \times 10^{-12} \mathrm{~F} / \mathrm{m}$ e $\mu_{0}=4 \pi \times 10^{-7} \mathrm{H} / \mathrm{m}$. As equações (1) e (2) possuem as seguintes soluções:

$$
\begin{aligned}
& \mathbf{E}=\mathbf{E}_{0} \exp \left[-i\left(\omega t \mp \mathbf{K} \cdot \mathbf{r}+\varphi_{E}\right)\right] \\
& \mathbf{B}=\mathbf{B}_{0} \exp \left[-i\left(\omega t \mp \mathbf{K} \cdot \mathbf{r}+\varphi_{B}\right)\right]
\end{aligned}
$$

sendo $\mathbf{E}_{0}$ a amplitude do campo elétrico, $\mathbf{B}_{0}$ a amplitude da indução magnética, $\omega=2 \pi \cdot c / \lambda$, a velocidade angular, $\mathbf{r}$ o vetor posição e $\mathbf{K}=2 \pi / \lambda$. $\hat{\mathbf{u}}$ o vetor de onda, $c$ a velocidade da luz no vácuo, $\lambda$ o comprimento da onda e $\hat{\mathbf{u}}$ o vetor unitário na direção de propagação. As constantes de fase associadas às soluções obtidas para o campo elétrico e magnético são, respectivamente, $\varphi_{E}$ e $\varphi_{B}$. As soluções encontradas representam ondas propagando-se da direita para a esquerda ou da esquerda para a direita, dependendo do sinal do vetor de onda $\mathbf{K}$.

No estudo da propagação da luz em meios materiais, o índice de refração é definido como a razão entre a velocidade da onda eletromagnética no vácuo e no meio material:

$n=\frac{c}{\mathrm{~V}}$

O índice de refração é uma grandeza complexa e está associado ao coeficiente de absorção pela expressão:

$\tilde{n}=n+i \frac{\alpha}{2} \frac{c}{\omega}$ 
$\mathrm{O}$ vetor de onda também pode ser escrito em função do coeficiente de absorção de acordo com:

$$
\mathbf{K}=n \frac{\omega}{c}+i \frac{\alpha}{2}
$$

A componente real do índice de refração apresenta uma dependência em relação ao comprimento de onda (ou energia). Esta dependência, conhecida como curva de dispersão do índice de refração, pode ser descrita a partir de uma equação empírica válida para qualquer material, pois seus parâmetros são obtidos do ajuste de dados experimentais. Na região de energia menor que o "gap" (região de transparência), este ajuste é obtido a partir da equação de Sellmeier (MONDRY et al., 1992):

$$
n(\lambda)^{2}=A+\frac{B \lambda^{2}}{\lambda^{2}-C^{2}}
$$

Na região de energia acima do "gap" (região de absorção), a componente real do índice de refração apresenta uma relação linear com o comprimento de onda, descrito pela expressão:

$$
n(\lambda)=a_{1}\left(\lambda_{g}-\lambda\right)+b_{1}
$$

sendo $a_{1}$ obtido do ajuste dos dados experimentais e $\mathrm{b}_{1} \mathrm{o}$ índice de refração no comprimento de onda da energia do "gap" $\left(\lambda_{g}\right)$, calculado a partir da equação de Sellmeier (MONDRY et al., 1992).

\section{Formalismo matricial}

Inicialmente consideremos um feixe de luz incidindo em uma interface que separa um meio material (índice 1) de outro meio material (índice 2). Utilizando as equações de Maxwell e aplicando condições de contorno adequadas, obtemos as relações entre a intensidade do campo elétrico e a indução magnética na interface. As componentes, na direção normal (índice $n$ ), do deslocamento elétrico
D e da indução magnética $\mathbf{B}$ são contínuas através da interface. Estas mesmas condições de contorno exigem que a componente tangencial (índice $t$ ) dos campos elétrico $\mathbf{E}$ e da intensidade magnética $\mathbf{H}$ também sejam contínuas através da superfície de separação entre os dois diferentes meios materiais:

$$
\begin{aligned}
& \mathbf{D}_{1 n}=\mathbf{D}_{2 n} \quad \rightarrow \quad \varepsilon_{1} \mathbf{E}_{1 n}=\varepsilon_{2} \mathbf{E}_{2 n} \\
& \mathbf{B}_{1 n}=\mathbf{B}_{2 n} \\
& \mathbf{H}_{1 t}=\mathbf{H}_{2 t} \quad \rightarrow \quad \frac{\mathbf{B}_{1 t}}{\mu_{1}}=\frac{\mathbf{B}_{2 t}}{\mu_{2}} \\
& \mathbf{E}_{1 t}=\mathbf{E}_{2 t}
\end{aligned}
$$

Consideremos o caso particular em que uma onda plana incide na direção perpendicular à interface, conforme o diagrama apresentado na Figura 1.

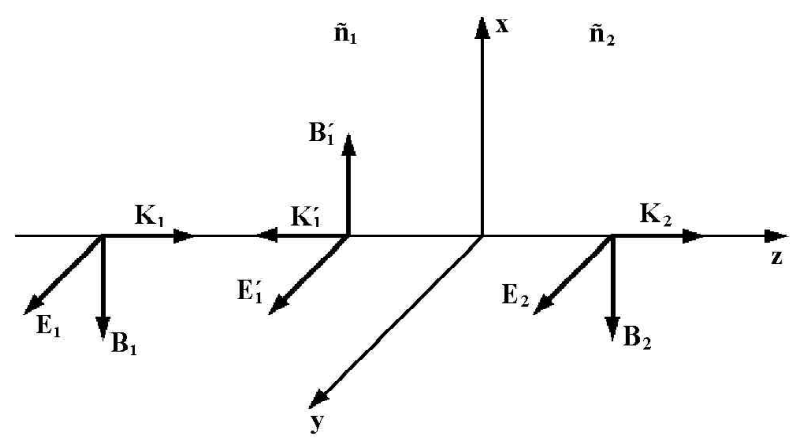

Figura 1 - Diagrama indicando as direções do campo elétrico $\mathrm{E}$, da inducão magnética $\mathrm{B}$ e do vetor de onda $\mathrm{K}$, para o caso particular de incidência normal.

Podemos escrever a dependência da intensidade do campo elétrico no meio material 2 em relação a intensidade do mesmo campo no meio material 1 , de acordo com:

$$
\mathbf{E}_{2}=\frac{2}{1+a_{12}} \mathbf{E}_{1} \equiv \tau_{12} \mathbf{E}_{1}
$$


$\operatorname{Com} a_{12}=\mathbf{K}_{2 \mathrm{z}} / \mathbf{K}_{1 \mathrm{z}}$, sendo as componentes $\mathbf{K}_{1 \mathrm{z}} \mathrm{e}$ $\mathbf{K}_{2 \mathrm{z}}$, as projeções dos vetores de onda $\mathbf{K}$ no meio material 1 e no meio material 2, respectivamente, na direção de propagação da onda. $\tau_{12}$ é o coeficiente de transmissão entre o meio material 1 e o meio material 2, definido como a razão entre a intensidade do campo elétrico da luz transmitida e a intensidade do campo elétrico da luz incidente. A dependência da intensidade do campo elétrico da luz refletida em relação ao campo elétrico incidente é dada por:

$\mathbf{E}_{1}^{\prime}=\frac{1-a_{12}}{1+a_{12}} \mathbf{E}_{1} \equiv \rho_{12} \mathbf{E}_{1}$

Sendo " $a$ " definido da mesma forma que para a equação (11) e o coeficiente de reflexão $\rho_{12}$ definido como a razão entre a intensidade do campo elétrico da luz refletida e a intensidade do campo elétrico da luz incidente. Estas expressões relacionando a intensidade do campo incidente com o refletido e transmitido, também são válidas para a indução magnética $\mathbf{B}$.

Consideremos agora, conforme descrito na Figura 2, um sistema de duas camadas de materiais com índices de refração diferentes. Nesta figura estão indicadas as direções de propagação de ondas eletromagnéticas se deslocando no interior do sistema. O campo elétrico total dentro de uma camada qualquer é composto por duas componentes, uma viajando da esquerda para a direita e outra viajando da direita para a esquerda. Cada camada possui duas interfaces com as camadas vizinhas, uma à sua direita e outra à sua esquerda.

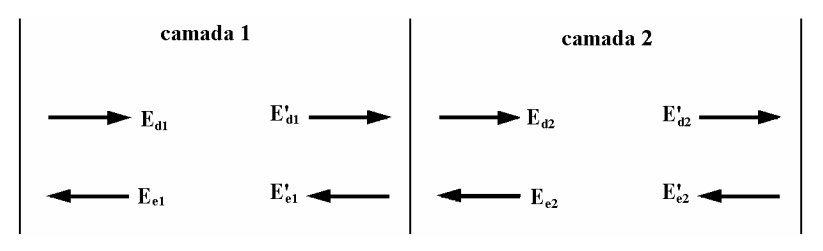

Figura 2 - Representação dos campos elétricos que descrevem as ondas eletromagnéticas viajando no interior de duas camadas arbitrárias de material dielétrico ou semicondutor em uma estrutura de multicamadas.
A mudança na intensidade da onda eletromagnética ao atravessar uma interface, é descrita pelo coeficiente de reflexão $\rho_{12}$ e pelo coeficiente de transmissão $\tau_{12}$, característicos da interface existente entre a camadas 1 e a camada 2 . O primeiro índice em subscrito na representação dos coeficientes se refere à camada inicial, de onde a onda está saindo, enquanto o segundo índice se refere à camada final do trajeto da onda. As relações entre a intensidade do campo elétrico nos dois lados de uma interface podem ser escritas como:

$\AA_{d 2}=\AA_{d 1}^{\prime} \hat{o}_{12}+\AA_{e 2} \tilde{n}_{21}$

$\mathbf{E}_{e 1}^{\prime}=\mathbf{E}_{e 2} \tau_{21}+\mathbf{E}_{d 1}^{\prime} \rho_{12}$

Combinando as duas equações acima, obtemos:

$\mathbf{E}_{e 1}^{\prime}=\mathbf{E}_{e 2}\left[\frac{\tau_{21} \cdot \tau_{12}-\rho_{21} \cdot \rho_{12}}{\tau_{12}}\right]+\mathbf{E}_{d 2} \frac{\rho_{12}}{\tau_{12}}$

Com a aplicação em (15) das relações de simetria entre os coeficientes de transmissão e reflexão, $\rho_{12}=-\rho_{21}$ e $\tau_{21} \tau_{12}+\left(\rho_{12}\right)^{2}=1$, obtemos para $o$ campo elétrico incidindo sobre a interface (lado direito da camada 1) a relação:

$\mathbf{E}_{d 1}^{\prime}=\left(\frac{\rho_{12}}{\tau}\right) \mathbf{E}_{e 2}+\left(\frac{1}{\tau}\right) \mathbf{E}_{d 2}$

Da mesma forma obtemos para o campo elétrico refletido pela interface, na camada 1 :

$\mathbf{E}_{e 1}^{\prime}=\left(\frac{1}{\tau}\right) \mathbf{E}_{e 2}+\left(\frac{\rho_{12}}{\tau}\right) \mathbf{E}_{d 2}$

As equações (16a) (16b) são equações lineares acopladas que descrevem a intensidade do campo elétrico da onda eletromagnética do lado esquerdo 
da interface em termos da intensidade do campo elétrico do lado direito. A partir destas equações é possível definir uma notação matricial para relacionar a intensidade dos campos ópticos de um feixe de luz que se propague através da interface entre as duas camadas. De acordo com a Figura 2, do lado esquerdo da camada 2 temos:

$$
\mathbf{E}_{2} \equiv\left(\begin{array}{l}
\mathbf{E}_{e 2} \\
\mathbf{E}_{d 2}
\end{array}\right)
$$

E do lado direito da camada 1, temos:

$$
\mathbf{E}_{1} \equiv\left(\begin{array}{l}
\mathbf{E}_{e 1}^{\prime} \\
\mathbf{E}_{d 1}^{\prime}
\end{array}\right)
$$

Portanto, as equações (16a) e (16b) podem ser expressas como uma operação de matrizes,

$$
\mathbf{E}_{1}=\mathbf{H}_{12} \mathbf{E}_{2}
$$

sendo $\mathbf{E}_{1}$ a matriz coluna apresentada em (17b), $\mathbf{E}_{2}$ a matriz coluna apresentada em (17a) e $\mathbf{H}_{12}$ a matriz de transição na interface. $\mathbf{H}_{12}$ é escrita em termos dos coeficientes de reflexão e transmissão, de acordo com:

$$
\mathbf{H}_{12} \equiv \frac{1}{\tau_{12}}\left(\begin{array}{cc}
1 & \rho_{12} \\
\rho_{12} & 1
\end{array}\right)
$$

É necessário ainda, descrever a mudança da intensidade do campo elétrico no interior de uma determinada camada. Para isto introduziremos o fator de fase, $\exp (-\mathrm{i} \beta), \operatorname{com} \beta$ definido por:

$$
\beta \equiv \frac{2 \pi}{\lambda} n d \cos \theta
$$

sendo $\lambda$ o comprimento da onda incidente (no vácuo), $\theta$ o ângulo de incidência do feixe de luz em relação à superfície, $n$ o índice de refração da camada e $d$ a espessura da camada. $\mathrm{O}$ fator de fase indica a variação da intensidade dos campos ópticos no interior da camada, gerando interferência destrutiva ou construtiva, de acordo com a relação entre a espessura da camada e o comprimento de onda.

$\mathrm{O}$ fator de fase permite obter a intensidade do campo elétrico de uma onda que chega em um lado da camada, em termos da intensidade da onda que saiu do outro lado da mesma camada. Para uma onda viajando da esquerda para a direita, a intensidade do campo elétrico é dada pela relação,

$\mathbf{E}_{d}^{\prime}=\mathbf{E}_{d} \cdot e^{-i \beta}$

Em uma onda se propagando da direita para a esquerda, na mesma camada, a intensidade do campo elétrico é dada por:

$\mathbf{E}_{e}^{\prime}=\mathbf{E}_{e} \cdot e^{i \beta}$

Com as equações (21a) e (21b) é possível relacionar através de uma expressão matricial a intensidade dos campos elétricos de um feixe de luz que se propague dentro de uma camada. Podemos descrever o campo do lado esquerdo da camada como função do campo do lado direito, de acordo com a expressão:

$\mathbf{E}=\mathbf{L}^{\mathbf{E}^{\prime}}$

Sendo $\mathbf{E}$ a matriz coluna representando o campo localizado no lado esquerdo da camada, $\mathbf{E}^{\prime}$ a matriz coluna representando o campo localizado no lado direito da camada e $\mathbf{L}$ a matriz propagação, escrita em termos do fator de fase, de acordo com:

$\mathbf{L} \equiv\left(\begin{array}{cc}e^{-i \beta} & 0 \\ 0 & e^{i \beta}\end{array}\right)$ 
Podemos aplicar este formalismo para estruturas compostas de um número maior de camadas de diferentes materiais, como é o caso dos espelhos de Bragg.

\section{Espelho de Bragg}

Espelho de Bragg são estruturas compostas por camadas alternadas de dois materiais com diferentes índices de refração, como descrito na Figura 3. Nesta figura, $n_{0}$ representa o índice de refração do ar, $n_{\mathrm{H}} \mathrm{o}$ índice de refração do meio material de maior índice, $n_{\mathrm{L}} \mathrm{o}$ índice de refração do meio material de menor índice e $n_{\mathrm{S}} \mathrm{o}$ índice de refração do substrato sobre o qual a estrutura de multicamadas foi preparada.

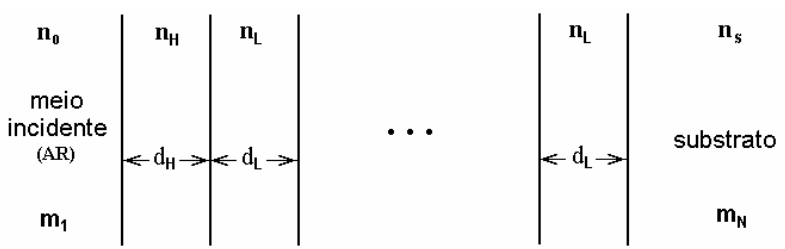

Figura 3 - Diagrama da estrutura de multicamadas denominada espelho de Bragg, preparada sobre um substrato. O índice de refração é indicado por $\mathrm{n}, \mathrm{d}$ representa a espessura da camada e $m_{i}$ representa, em ordem crescente, os diversos meios de propagação da luz, a partir do meio de incidência (o ar).

Podemos identificar, no diagrama apresentado, uma unidade que se repete, formada por um par de camadas com índice de refração alto e baixo. Este par de camadas pode ser descrito na notação matricial por:

$$
\mathbf{S}_{\mathrm{par}}=\mathbf{H}_{\mathrm{LH}} \mathbf{L}_{\mathrm{H}} \mathbf{H}_{\mathrm{HL}} \mathbf{L}_{\mathrm{L}}
$$

Sendo,

$$
\mathbf{H}_{H L}=\frac{1}{\tau_{H L}}\left(\begin{array}{cc}
1 & \rho_{H L} \\
\rho_{H L} & 1
\end{array}\right)
$$

$$
\mathbf{H}_{L H}=\frac{1}{\tau_{L H}}\left(\begin{array}{cc}
1 & \rho_{L H} \\
\rho_{L H} & 1
\end{array}\right)
$$

A matriz $\mathbf{H}_{H L}$ representa a transição da camada com índice de refração maior $\left(n_{H}\right)$ para a camada com índice de refração menor $\left(n_{L}\right)$, e $\mathbf{H}_{L H}$ representa a transição da camada com índice de refração menor $\left(n_{L}\right)$ para a camada com índice de refração maior $\left(n_{H}\right)$. Os termos $\mathbf{L}_{H}$ e $\mathbf{L}_{L}$ representam a matrizes propagação no material com índice de refração $n_{H} \mathrm{e}$ $n_{L}$, respectivamente:

$$
\mathbf{L}_{H}=\left(\begin{array}{cc}
e^{-i \beta_{H}} & 0 \\
0 & e^{i \beta_{H}}
\end{array}\right)
$$

$$
\mathbf{L}_{L}=\left(\begin{array}{cc}
e^{-i \beta_{L}} & 0 \\
0 & e^{i \beta_{L}}
\end{array}\right)
$$

As matrizes indicadas em (24b e 24c), podem ser reescritas com o auxilio das equações (11) e (12):

$$
\mathbf{H}_{H L}=\frac{1}{2 K_{H}}\left(\begin{array}{ll}
K_{H}+K_{L} & K_{H}-K_{L} \\
K_{H}-K_{L} & K_{H}+K_{L}
\end{array}\right),
$$

$$
\mathbf{H}_{L H}=\frac{1}{2 K_{L}}\left(\begin{array}{ll}
K_{L}+K_{H} & K_{L}-K_{H} \\
K_{L}-K_{H} & K_{L}+K_{H}
\end{array}\right)
$$

As matrizes indicadas em (25a e 25b), podem ser reescritas com o auxílio da equação (20),

$$
\mathbf{L}_{H}=\left(\begin{array}{cc}
e^{-i K_{H} \cdot d_{H}} & 0 \\
0 & e^{i K_{H} \cdot d_{H}}
\end{array}\right) \quad \mathbf{L}_{L}=\left(\begin{array}{cc}
e^{-i K_{L} \cdot d_{L}} & 0 \\
0 & e^{i K_{L} \cdot d_{L}}
\end{array}\right)
$$

A grande espessura do substrato em relação à espessura total do espelho de Bragg faz com que o substrato absorva totalmente a onda eletromagnética incidente. Isto leva à uma condição de contorno que 
implica na não existência de onda eletromagnética, dentro do substrato, se deslocando da direita para a esquerda. Para descrever o espelho de Bragg é necessário aplicar esta condição de contorno, de modo a obter o ponto inicial de construção da matriz que representa a estrutura. Portanto, a matriz coluna que representa a intensidade dos campos no lado esquerdo do substrato é escrita como:

$$
\mathbf{E}_{S}=\left(\begin{array}{c}
0 \\
E_{d S}
\end{array}\right)
$$

Para descrever a estrutura completa do espelho de Bragg, realizamos a multiplicação de matrizes representando as transições de todas as camadas obtendo:

$$
\mathbf{E}_{\text {total }}=\mathbf{H}_{a r H} \mathbf{L}_{H}\left(\mathbf{S}_{p a r}\right)^{\mathrm{N}} \mathbf{E}_{\mathrm{s}}
$$

O produto de matrizes $\mathbf{S}_{\text {total }}=\mathbf{H}_{a r H} \mathbf{L}_{H}\left(\mathbf{S}_{p a r}\right)^{\mathrm{N}}$ contém os efeitos combinados de todas as camadas incluindo as múltiplas reflexões. Podemos construir $\mathbf{S}_{\text {total }}$ para um número qualquer de camadas se conhecermos individualmente o índice de refração e a espessura das camadas, o coeficiente de reflexão e o coeficiente de transmissão característicos das interfaces entre as camadas dos diferentes materiais que constituem a estrutura.

A intensidade do campo elétrico $\left(\mathbf{E}_{\text {total }}\right)$ de um feixe de luz refletido e transmitido pelo espelho de Bragg pode ser obtida a partir das relações entre a intensidade do campo elétrico no meio material incidente (ar) e no meio material final (substrato):

$\mathbf{E}_{\text {total }}=\mathrm{S}_{\text {total }} \mathbf{E}_{\mathrm{s}}$

Escrevendo a operação de matrizes de forma mais explícita, temos:
$\left(\begin{array}{c}\mathbf{E}_{\text {etotal }} \\ \mathbf{E}_{d_{\text {total }}}\end{array}\right)=\left(\begin{array}{ll}S_{11} & S_{12} \\ S_{21} & S_{22}\end{array}\right)\left(\begin{array}{c}0 \\ \mathbf{E}_{d S}\end{array}\right)$

E portanto:

$\left(\begin{array}{c}\mathbf{E}_{\text {e total }} \\ \AA_{\text {d total }}\end{array}\right)=\left(\begin{array}{l}S_{12} E_{d S} \\ s_{22} E_{d S}\end{array}\right)$

A relação entre a intensidade do campo elétrico refletido pela estrutura $\left(\mathbf{E}_{e \text { total }}\right)$ dividido pela intensidade do campo elétrico incidente $\left(\mathbf{E}_{d \text { total }}\right)$, é definida como o coeficiente de reflexão da estrutura:

$R=\frac{E_{\text {etotal }}}{E_{d \text { total }}}=\frac{S_{12} E_{d S}}{S_{22} E_{d S}}=\frac{S_{12}}{S_{22}}$

O coeficiente de transmissão da estrutura é definido pela razão entre a intensidade do campo elétrico da onda transmitida através do substrato $\left(\mathbf{E}_{d S}\right)$ e a intensidade do campo elétrico da onda incidente $\mathbf{E}_{\text {d total }}$ :

$T=\frac{E_{d S}}{E_{d \text { total }}}=\frac{E_{d S}}{S_{22} E_{d S}}=\frac{1}{S_{22}}$

O espelho apresentará reflexão máxima se as espessuras das camadas forem tais que a multiplicação do índice de refração com a espessura, para cada camada, seja igual a um quarto do comprimento de onda. Nesta condição, teremos uma situação que resultará em interferência construtiva na saída do sinal óptico para o ar.

A partir da matriz que representa o coeficiente de reflexão (30), pode-se realizar o cálculo da refletividade em espelho de Bragg (KLEIN; FURTAK, 1986) através de uma rotina utilizando cálculo numérico, utilizando a seguinte expressão:

$\mathrm{r}=|R|^{2}$ 
Para desenvolver o programa de simulação necessitamos das propriedades ópticas dos diferentes materiais que compõem a estrutura do espelho de Bragg. Estas propriedades são o índice de refração, coeficiente de absorção e energia de gap. No próximo item faremos uma revisão destas propriedades para os materiais que compõem o espelho de Bragg mostrado neste trabalho.

\section{Sistema AlGaAsSb/AlAsSb sobre substrato de} InP

Materiais da família do antimônio são muito promissores para o desenvolvimento de espelhos de Bragg para a região de $1,55 \mu \mathrm{m}$. Estes materiais apresentam grande constraste dos índices de refração ( $\Delta n \cong 0,54$ para $\mathrm{AlGaAsSb} / \mathrm{AlAsSb}$ ), o que permite a preparação de espelhos de Bragg de alta refletividade com um número pequeno de camadas.

A energia de "gap" direto (vale $\Gamma$ da primeira zona de Brillouin) para a liga $\mathrm{Al}_{\mathrm{x}} \mathrm{Ga}_{1-\mathrm{x}} \mathrm{As}_{\mathrm{y}} \mathrm{Sb}_{1-\mathrm{y}}$ à temperatura de $300 \mathrm{~K}$ pode ser obtida através de um método de interpolação (BENJAMIN, 2000).

$$
\begin{aligned}
\mathrm{E}_{\mathrm{g}}^{\tilde{\mathrm{A}}}(\mathrm{x}, \mathrm{y}) & =0,72+1,11 \mathrm{x}-0,5 \mathrm{y}+1,25 \mathrm{xy} \\
& +0,47 \mathrm{x}^{2}+1,2 \mathrm{y}^{2}-0,1 \mathrm{x}^{2} \mathrm{y}-1,2 \mathrm{y}^{2} \mathrm{x}
\end{aligned}
$$

Sendo $x$ a composição relativa dos materiais tipo III e $y$ a composição relativa dos materiais tipo V. Na expressão (33), os parâmetros $x$ e $y$ podem variar de 0 a 1.

A liga $\mathrm{Al}_{\mathrm{x}} \mathrm{Ga}_{1-\mathrm{x}} \mathrm{As}_{\mathrm{y}} \mathrm{Sb}_{1-\mathrm{y}}$ em condição de rede casada com o InP, com valores de $x$ menores que 0,45 possui "gap" direto (vale $\Gamma$ ). Para valores de $x$ maiores, o "gap" é indireto, sendo, a banda $X$ a de menor energia (vale $X$ da primeira zona de Brillouin). O casamento do parâmetro de rede da liga quaternária $\mathrm{Al}_{\mathrm{x}} \mathrm{Ga}_{1-\mathrm{x}} \mathrm{As}_{\mathrm{y}} \mathrm{Sb}_{1-\mathrm{y}}$ com o substrato de InP ocorre para o intervalo de concentrações entre 0,513 e 0,5622 (elementos do grupo V).

A dispersão do índice de refração real da liga
$\mathrm{Al}_{\mathrm{x}} \mathrm{Ga}_{1-\mathrm{x}} \mathrm{As}_{\mathrm{y}} \mathrm{Sb}_{1-\mathrm{y}}$, pode ser descrita pelo modelo baseado na equação de Sellmeier (MONDRY et al., 1992). Para uma liga com concentração de $13 \%$ de alumínio (JEANNES, 1996), a equação (8) é escrita como:

$n_{1}=\left(12,8+\frac{0,19 \lambda^{2}}{\lambda^{2}-1140^{2}}\right)^{1 / 2}$

sendo $n_{1}$ o índice de refração real para a região de transparência, com $\lambda$ dado em nm. A curva de dispersão do índice de refração real para a região de absorção de acordo com a expressão (9) é escrita como (JEANNES, 1996):

$$
n_{1 b}=86 \times 10^{-6}\left(\frac{1,24}{E_{g}(x, y)}-\lambda\right)+n_{1}\left(E_{g}(x, y)\right)
$$

Assim, a componente real do índice de refração é escrita como:

$n=n_{1}+n_{1 b}$

O comportamento da componente imaginária, expressão (6), do índice de refração é descrita pelo coeficiente de absorção, que, para a liga $\mathrm{Al}_{x} \mathrm{Ga}_{1-}$ ${ }_{x} \mathrm{As}_{\mathrm{y}} \mathrm{Sb}_{1-\mathrm{y}}$ tem o valor aproximado de $1,3 \times 10^{-4}$ [NABET, 1997].

O cálculo do "gap" para a liga ternária $\mathrm{AlAs}_{\mathrm{y}} \mathrm{Sb}_{1-\mathrm{y}}$ em condição de rede casada com o InP , é obtido a partir da equação (BENJAMIN, 2000):

$$
E_{g}(y)=1,61+0,55 y
$$

O "gap" desta liga é indireto, sendo a banda $X$ a banda de menor energia, para todo valor de $y$. A condição de rede casada com o InP é obtida para $y \cong$ 0,562 (BENJAMIN, 2000). 
A curva de dispersão para a componente real do índice de refração na forma da equação (9), é descrita por (JEANNES, 1996):

$$
n(\lambda)=86 \times 10^{-6} \times(1550-\lambda)+3,12
$$

sendo $\lambda$ o comprimento de onda dado em $\mathrm{nm}$.

O InP, utilizado como substrato na preparação do espelho, apresenta "gap" direto. O valor do "gap" é $1,423 \mathrm{eV}$ à temperatura de $4,4 \mathrm{~K} \mathrm{e} 1,34 \mathrm{eV}$ à $300 \mathrm{~K}$ (MADELUNG, 1996). Na região espectral entre $1000 \mathrm{~nm}$ e $2000 \mathrm{~nm}$, o InP é transparente, apresentando um coeficiente de absorção muito pequeno, da ordem de $1,7 \times 10^{-3}$. A curva de dispersão do índice de refração do InP também pode ser descrita pela equação de Sellmeier (SEMICONDUCTORS AND SEMIMETALS, 1967):

$$
n_{1}=\left(7,255+\frac{2,316 \lambda^{2}}{\lambda^{2}-626,56^{2}}\right)^{1 / 2}
$$

\section{Detalhes experimentais e de cálculo}

Utilizamos neste trabalho uma amostra de espelho de Bragg preparada pela da técnica de epitaxia por feixe molecular-MBE (Molecular Beam Epitaxy). As características da amostra estão indicadas na Tabela 1. A amostra foi preparada sobre substrato de InP tipo $n$, na direção cristalográfica (100). A dopagem por Telúrio foi efetuada utilizando-se como fonte $\mathrm{o}$ $\mathrm{Sb}_{2} \mathrm{Te}_{3}$. A composição dos materiais foi escolhida para obter transparência em $1.55 \mu \mathrm{m}$. As medidas de refletividade foram obtidas através de um espectrômetro FTIR (Fourier Transform Infra-Red), na configuração VW (PALMER, 1995). Nesta técnica, um primeiro espectro é obtido com uma reflexão simples em um espelho. Este primeiro espectro é normalizado para o máximo valor da refletividade obtido, através da chamada configuração VW. Esta configuração requer duas reflexões em dois pontos diferentes da amostra. Isto permite obter uma medida absoluta da refletividade, mas o espectro pode ser afetado se as camadas não forem uniformes sobre toda a amostra. A técnica utilizada proporcionou uma medida absoluta com uma precisão da ordem de $0.2 \%$ (PALMER, 1995) para a forma do espectro de refletividade .

Tabela 1 - Descrição nominal da amostra 66N24.

\begin{tabular}{|c|c|c|c|c|c|c|}
\hline Amostra & $\begin{array}{c}\text { Número de } \\
\text { períodos }\end{array}$ & Materiais & \multicolumn{2}{|c|}{ Compos ição } & $\begin{array}{c}\text { Dopagem } \\
\left(10^{18} \mathrm{~cm}^{-3}\right)\end{array}$ & $\begin{array}{c}\text { Espessura } \\
\text { da camada (nm) }\end{array}$ \\
\cline { 4 - 5 } & 20,5 & $\mathrm{Al}_{\mathrm{x}} \mathrm{Ga}_{1-\mathrm{x}} \mathrm{As}_{\mathrm{y}} \mathrm{Sb}_{1-\mathrm{y}}$ & 0.12 & 0.51 & 5,0 & 118 \\
\cline { 3 - 5 } & & $\mathrm{AsAs}_{\mathrm{y}} \mathrm{Sb}_{1-\mathrm{y}}$ & - & 0.56 & 3,0 & 102 \\
\hline
\end{tabular}

Para elaborarmos o programa de simulação escolhemos como ferramenta o aplicativo Mathcad 6.0. Tal escolha se deve ao fato do aplicativo ser capaz de resolver os cálculos necessários de forma satisfatória e apresentar uma interface prática e amigável, sendo ainda possível importar tabelas de dados experimentais para comparar com os dados calculados e também exportar os dados gerados em forma de tabelas para utilizá-los em outro aplicativo. O aplicativo também possibilita a elaboração de gráficos com rapidez, permitindo visualizar graficamente com facilidade as expressões usadas nas diferentes etapas do cálculo (apêndice 1). 


\section{Discussão e conclusões}

$\mathrm{Na}$ Figura 4 apresentamos os dados experimentais da medida de refletividade da amostra 66N24 e o espectro obtido por simulação:

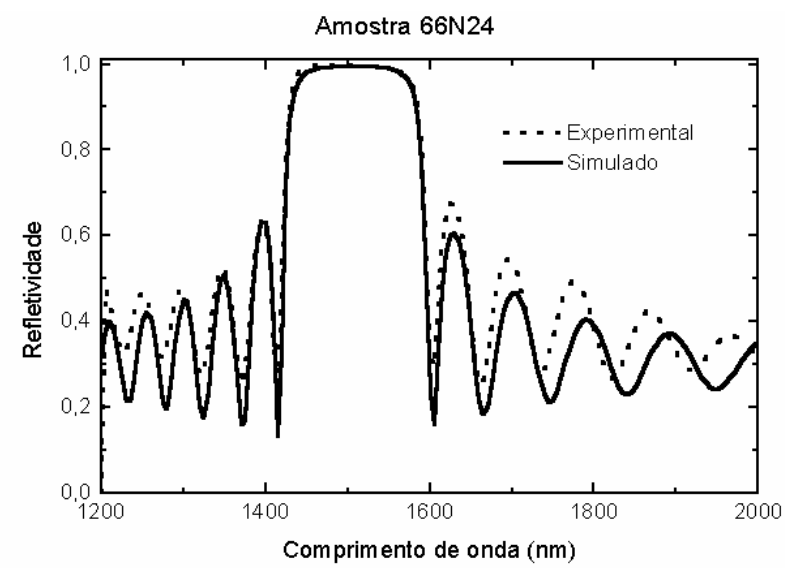

Figura 4 - Refletividade experimental e calculada por simulação para a amostra $66 \mathrm{~N} 24\left(\mathrm{Al}_{0},{ }_{12} \mathrm{Ga}_{0,88} \mathrm{As}_{0,51} \mathrm{Sb}_{0,49}\right)$.

Na Tabela 2 apresentamos algumas características ópticas do espelho de Bragg de AlGaAsSb: a espessura da camada de $\mathrm{AlGaAsSb}\left(d_{\mathrm{H}}\right)$, a espessura da camada de $\mathrm{AlAsSb}\left(d_{\mathrm{L}}\right)$, a diferença entre o índice de refração da liga ternária e quaternária $(\Delta n)$, a refletividade máxima $(\mathrm{R})$, o comprimento de onda central $(\lambda c)$, a largura do "stop band" $(\Delta \lambda)$.
Observamos que mesmo com um número relativamente pequeno de períodos (20,5), obtivemos uma alta refletividade, quando comparado a outros sistemas, como InGaAsP/InP ou AlGaInAs/AlInAs (DIAS et al., 1998). O número pequeno de períodos permite a preparação de espelhos de Bragg com menor espessura e com menor tempo de crescimento. A espessura total do espelho de Bragg estudado é de $4,518 \mu \mathrm{m}$. Este é um valor inferior ao utilizado em espelhos de Bragg com a mesma refletividade fabricados com ligas de outros materiais semicondutores como por exemplo InGaAsP/InP (DIAS et al., 1998). Devido ao menor contraste do índice de refração, para se obter uma refletividade de $\Delta n \cong 0,27$ na liga InGaAsP/InP, é necessário preparar o espelho de Bragg com 40,5 períodos, levando a uma espessura total de 9,472 $\mu \mathrm{m}$. Estruturas com espessuras muito grandes podem trazer dificuldades para a etapa de processamento dos dispositivos. A grande largura da "stop band" também é consequiência do grande contraste do índice de refração, possível de obter com os materiais da família do antimônio (ver Tabela 2). A dopagem com $\mathrm{Te}$, apesar de relativamente alta (Tabela 1) não parece ter afetado de forma significativa a refletividade.

Tabela 2 - Características ópticas da amostra 66N24:

\begin{tabular}{|c|c|c|c|c|c|c|c|c|}
\hline \multirow{2}{*}{ Amostra } & \multicolumn{2}{|c|}{$d_{\mathrm{H}}(\mu \mathrm{m})$} & \multicolumn{2}{|c|}{$d_{\mathrm{L}}(\mu \mathrm{m})$} & $\Delta n$ & $\mathrm{R}$ & $\lambda_{\mathrm{C}}(\mu \mathrm{m})$ & $\Delta \lambda(\mu \mathrm{m})$ \\
\hline \multirow{2}{*}{ 66Q24 } & nominal & 0,1180 & nominal & 0,1020 & \multirow{2}{*}{0,54} & 0,998 & 1,510 & 0,190 \\
\cline { 2 - 9 } & Simulado & 0,1190 & simulado & 0,1040 & & & & \\
\hline
\end{tabular}

O espectro da refletividade, obtido por simulação, se ajusta bem aos dados experimentais na região de menor comprimento de onda, com os máximos e mínimos secundários da refletividade mostrando excelente coincidência. Entretanto, na região de maiores comprimentos de onda observase que o espectro de refletividade calculado não apresenta boa concordância com os dados experimentais. Acreditamos que estas discrepâncias podem estar relacionadas ao ainda relativo desconhecimento das propriedades ópticas dos materiais da família do antimônio (AlGaAsSb/ AlAsSb), utilizados na preparação do espelho de Bragg. Estes materiais ainda não foram estudados 
de modo tão sistemáticos quanto outros materiais como os da família do fósforo. Outra possível fonte de erro encontra-se nos valores obtidos para a composição da liga quaternária que podem oscilar um pouco em relação aos valores nominais apresentados. Não consideramos ainda os efeitos que a dopagem, em uma concentração relativamente alta $\left(3 \times 10^{18} \mathrm{~cm}^{-3}\right.$ a $\left.5 \times 10^{18} \mathrm{~cm}^{-3}\right)$, poderia apresentar sobre o índice de refração dos materiais. Entretanto, os resultados obtidos mostram de forma geral uma boa concordância da simulação com os dados experimentais. Trabalhos mais sistemáticos estão sendo desenvolvidos visando melhorar o conhecimento das propriedades ópticas dos materiais da família do antimônio. Devido aos resultados obtidos neste trabalho, como a alta refletividade, grande largura da "stop band" para um número relativamente pequeno de períodos e o pequeno efeito da dopagem sobre o valor da refletividade podemos afirmar que as ligas dos materiais $\mathrm{AlGaAsSb} / \mathrm{AlAsSb}$ surgem como boas candidatas para a preparação de espelhos de Bragg em lasers verticais na região de grandes comprimentos de onda.

\section{Referências}

BENJAMIN, E.A . Determinação da energia de “"gap”' de materiais semicondutores da família do antimônio . 2000. Monografia (Bacharel em Física) Universidade Estadual de Londrina, Londrina.

DIAS, I.F.L. et al. Determinação de parâmetros de ligas ternárias e quaternárias da família do antimônio. Londrina: Universidade Estadual de Londrina, 1996.

Nota Técnica do Grupo de Óptica e Optoeletrônica, n. 1.

DIAS, I.F.L. et. al. Electrical and Optical Characteristics of n-type doped Distributed Bragg Mirrors. IEEE Photon. Tech. Letters, v.10, n.6, p.763$765,1998$.

DIAS, I.F.L. et al. High Reflectivity and Low resistance Te doped AlGaAsSb/AlAsSb Bragg mirror. Electr. Letters, v.33, n.8, p.716-717, 1997.
EBELING, K.J. Optical interconnects and data links with Vertical Surface Emitting Lasers Diodes(VCSEL). ECOC's , 21 st , 1995, Bruxelas. Eur. Conf. On Opt. Comm. Proceedings... Bruxelas,1995. p.113.

GIEHLER, M. et al. Optical properties of lowtemperature groww GaAs on Bragg reflectors. Journal of Applied Physics, v.92, n.6, p.2974, 2002.

HARMAND, J.C. et al. AlAsSb/AlGaAsSb Bragg stacks for $1.55 \mathrm{~mm}$ wavelength grown by molecular beam epitaxy. Electr. Letters, v.31, n.19, p.1689-1690, 1995.

HARMAND, J.C.; KOHL, A.; JUHEL, M.; LEROUX, G. Molecular Beam Epitaxy of AlGaAsSb system for $1.55 \mathrm{~mm}$ Bragg mirrors. J. Crystal Growth, v.175, p.372-376, 1997.

JEANNES, F. et al. Dispositifs bistables à cavitéverticale pour commutation tout-optique aux longueurs d'onde télécom. 1996. Tese (Doutorado em Ciências) Université D'Aix-Marseille III, Marseille.

KLEIN, M. V.; FURTAK, T. E. Optics. New York: John Wiley \& Sons, 1986.

MADELUNG, O. Semiconductors - Basic Data. 2.ed. Berlin: Springer-Verlag, 1996.

MONDRY, M.J. et al. Refractive Indexes of (Al, Ga, In)As Epilayers in InP for Optoelectronic Applications . IEEE Photonics Technology Letters, v.4, n.6, p.627630, 1992.

NABET, B. Comunicação interna CNRS URA 250, Laboratoire CDP. Bagneux, 1997.

NAKAGAWA, S. et al. 1.55 ?m InP-Lattice -Matched VCSELs with AlGaAsSb-AlAsSb DBRs. IEEE Journal on Selected Topics in Quantum Electronics, v.7, n.2, p.224, 2001.

PALMER, J. M. The Measurements of transmission, absorption, emission, and reflexion, Handbook of Optics Part-II. New York: McGraw-Hill, 1995.

REITZ, J. R.; MILFORD, F. J.; CHRISTY, R. W. Fundamentos da Teoria Eletromagnética. Rio de Janeiro: Ed. Campus, 1982.

SALE, T.E. Vertical Cavity Surface Emitting Lasers. New York: John Wiley \& Sons, 1995.

SEMICONDUCTORS AND SEMIMETALS. Optical Properties of III-V Compounds. New York: Academic Press, 1967. v.3, p.528. 


\section{Apêndice 1}

Fluxograma de simulação para espelhos de Bragg AlGaAsSb/AlAsSb

\section{Definições gerais}

- Definição da equação (8) - Dispersão do índice de refração real na região de transparência $\operatorname{Sellmeier} 1(A, B, C, \lambda)=\sqrt{A+\frac{B \cdot \lambda^{2}}{\lambda^{2}-C^{2}}}$

- Definição da equação (9) - Dispersão do índice de refração na região de absorção

Sellmeier $2(a 1, b 1, \lambda g, \lambda)=a_{1} \cdot\left(\lambda_{g}-\lambda\right)+b_{1}$

- Definição da relação de conversão energia (eV) para comprimento de onda (nm)

$\operatorname{comp} \lambda(E)=\frac{1,2398}{E \cdot 10^{-3}}$

- Definição da matriz transição de interface transição aa camada 1 para a camada 2

$$
M \operatorname{int}(k 1, k 2)=\frac{1}{2 k 1 z}\left[\begin{array}{cc}
(k 1+k 2) & (k 1-k 2) \\
(k 1-k 2 z) & (k 1+k 2)
\end{array}\right]
$$

- Definição da matriz de propagação em uma camada 1, com espessura e1 e vetor de onda K1.

$$
\operatorname{Mprop}(e 1, k 1):=\left[\begin{array}{cc}
e^{i k 1 . e 1} & 0 \\
0 & e^{-i k 1 . e 1}
\end{array}\right]
$$

- Definição da matriz da célula unitária - composta por um par de camadas 1 e camada 2

$\operatorname{Mcel}(\mathrm{k} 1, \mathrm{e} 1, \mathrm{k} 2, \mathrm{e} 2)=$ $\operatorname{Mint}(\mathrm{k} 1, \mathrm{k} 2) \cdot \operatorname{Mprop}(\mathrm{e} 2, \mathrm{k} 2) \cdot \operatorname{Mint}(\mathrm{k} 2, \mathrm{k} 1) \operatorname{Mpro}(\mathrm{e} 1, \mathrm{k} 1)$
- Definição da matriz total do espelho de Bragg $\operatorname{MBragg}(\mathrm{k} 1, \mathrm{e} 1, \mathrm{k} 2, \mathrm{e} 2, \mathrm{kar}, \mathrm{ksub}, \mathrm{n})=$ $\operatorname{Mint}(k a r, k 1) \cdot M p r o p(e 1, k 1) \cdot M c e l(k 1, e 1, k 2, e 2)^{\mathrm{n}}$. $\operatorname{Mint}(\mathrm{k} 1, \mathrm{ksub})$

\section{Propriedades estruturais e ópticas dos meios materiais:}

- Definição do índice de refração do ar $\operatorname{nar}(\lambda)=1$

\section{Propriedades da liga InP}

- Definição do índice de refração real na região de transparência (MADELUNG, 1996)

nrealIn $P(A, B, C, \lambda):=n 1(7.255,2.316,626.26, \lambda)$

- Definição do coeficiente de absorção (MADELUNG, 1996)

$\alpha \operatorname{In} P(\lambda)=2.10^{-3}$

- Definição da componente imaginária do índice de refração (equação 6)

$\operatorname{nimag} \operatorname{In} P=\frac{\alpha \operatorname{In} P(\lambda)}{2} \cdot \frac{\lambda}{2 \pi}$

para $\lambda \leq \lambda g \operatorname{In} P$

- Definição do índice de refração complexo $\operatorname{ncomplex} \operatorname{InP}=\operatorname{nrealInP}(\lambda)-i \cdot \operatorname{nimag} \operatorname{Inp}(\lambda)$ 


\section{Propriedades da liga AIGaAsSb}

- Definição da energia de "gap" em função da composição da liga (equação 33)

EgAlGaAs $\operatorname{Sb}(\mathrm{x}, \mathrm{y})=0,72+1,11 \mathrm{x}-0,5 \mathrm{y}+1,25 \mathrm{xy}+0,47 \mathrm{x}^{2}$

$$
+1,2 y^{2}-0,1 x^{2} y-1,2 y^{2} x
$$

EgapAlGaAs $S b=E g A l G a A s S b(0.13,0.513)$

- Definição do comprimento de onda, na energia de "gap"

$\lambda$ gapAlGaAs $S b:=\operatorname{comp} \lambda($ Egap AlGaAs $S b)$

- Definição do índice de refração real, na região de transparência (JEANNES, 1996)

$n r 1 A l G a A s \operatorname{Sb}(\lambda):=$ Sellmeier $1(12.8,0.19,1140, \lambda)$

- Definição do índice de refração real na energia de "gap"

b1 AlGaAs $S b=n r 1 A l G a A s S b(\lambda g a p A l G a A s S b)$

- Definição do índice de refração real, na região de absorção

$n r 2 A l G a A s S b(\lambda):=\operatorname{Sellmeier} 2\left(86 \cdot 10^{-6}, b 1 A l G a A s S b, \lambda\right.$ gapAlGaAs $\left.S b, \lambda\right)$

- Definição do índice de refração real total nrealAlGaAs $S b(\lambda)=n r 1 A l G a A s S b$ para $\lambda \geq \lambda$ gap AlGaAs $S b$ nrealAlGaAs $S b(\lambda)=n r 2 A l G a A s S b$ para $\lambda \leq \lambda g$ AlGaAs $S b$
- Definição do coeficiente de absorção (NABET, 1997)

$$
\alpha A l G a A s \operatorname{Sb}(\lambda)=1.3 \cdot 10^{-4}
$$

- Definição da componente imaginária do índice de refração

$$
\operatorname{nimagAlGaAs} S b(\lambda)=\frac{\alpha A l G a A s S b}{2} \cdot \frac{\lambda}{2 \pi}
$$

para $\quad \lambda \leq \lambda$ gap AlGaAs $S b$

nimagAlGaAs $S b=0$

para $\quad \lambda \geq \lambda$ gap AlGaAs Sb

- Definição do índice de refração complexo ncomplexo AlGaAs $S b:=$ nrealAlGaAs $S b-i \cdot$ nimagAlGaAs $S b$

\section{Propriedades da liga AlAsSb}

- Definição da energia de "gap" em função da composição da liga

EgAlAs $S b(y)=1.61+0.55 y$

EgapAlAs $S b=$ EgAlAs $S b(0.56)$

- Definição do comprimento de onda na energia de "gap"

$\lambda$ gap AlAs $S b=\lambda E($ EgapAlAs $S b)$

- Definição do índice de refração real (não dispomos dos parâmetros para a equação de Sellmeier1)

$n r 2 A l A s \operatorname{Sb}(\lambda):=$ Sellmeier $2\left(86 \cdot 10^{-6}, 3.21, \lambda\right.$ gapAlAs $\left.S b, \lambda\right)$ 
- Componente imaginária do índice de refração (não dispomos do coeficiente de absorção)

- Índice de refração complexo da liga $\mathrm{AlAsSb}$ ncomplexo AlAs $S b=$ nrealAlAs $S b$

\section{Cálculo do espelho de Bragg de AlGaAsSb/} AlAsSb :

- Definição do comprimento de onda central (em $\mathrm{nm})$

br: $\quad 1502$

- Definição do número de períodos inteiros do espelho

nperiod $=20$

- Definição da espessura da camada da liga $\mathrm{AlGaAsSb}(\mathrm{em} \mathrm{nm})$

ealGaAsSb=104

- Definição da espessura da camada da liga AlAsSb (em nm)

ealAsSb:=119

- Definição do vetor de onda no vácuo $k 0(\lambda)=\frac{2 \pi}{\lambda}$
- Definição do vetor de onda no ar $k 0(\lambda)=k 0(\lambda) \cdot \sqrt{\operatorname{nar}(\lambda)^{2}}$

- Definição do vetor de onda na liga AlGaAsSb $k A l G a A s S b(\lambda)=k o(\lambda) \cdot \sqrt{n c o m p l e x o A l G a A s S b(\lambda)^{2}}$

- Definição do vetor de onda na liga AlAsSb

$$
k A l A s S b(\lambda)=k o(\lambda) \cdot \sqrt{n \text { complexoAlAsSb( } \lambda)^{2}}
$$

- Definição do vetor de onda na liga InP

$$
k \operatorname{In} P(\lambda)=k o(\lambda) \cdot \sqrt{n \text { complexoInP( }(\lambda)^{2}}
$$

- Cálculo do coeficiente de reflexão (a partir das componentes da matriz na equação 30)

$R(\lambda)=\frac{\operatorname{Mbragg}(k A l G a A s S b(\lambda), e A l G a A s S b, k A l A s S b(\lambda), e A l A s S b, k a r(\lambda), k \operatorname{InP}(\lambda), \text { nperiod })_{1,2}}{\operatorname{Mbragg}(k A l G a A s S b(\lambda), e A l G a A s S b, k A l A s S b(\lambda), e A l A s S b, k a r(\lambda), k \operatorname{In} P(\lambda), \text { nperiod })_{2,2}}$

- Cálculo da Refletividade (de acordo com a equação 32)

$$
\operatorname{Rm}(\lambda):=|R(\lambda)|^{2}
$$

- Escrever o resultado da simulação em forma de tabela

- Exportar os dados da tabela para um arquivo do tipo asc 
